\title{
Facial myokymia: a clinicopathological study
}

\author{
P. K. SETHI ${ }^{1}$, BERNARD H. SMITH, AND K. KALYANARAMAN \\ From the Department of Neurology, Edward J. Meyer Memorial Hospital \\ and School of Medicine, State University of New York at Buffalo, N.Y., U.S.A.
}

SYNOPSIS Clinicopathological correlations are presented in a case of facial myokymia with facial palsy. The causative lesions were considered to be metastatic tumours to the pons and it was concluded that both the facial palsy and the myokymia were due to interruption of supranuclear pathways impinging on the facial nucleus.

Oppenheim (1916) described a patient with continuous undulation and fasciculation in the right facial muscles. The movements had started in the infraorbital region and progressed to involve the entire territory of the facial nerve. He called the condition facial myokymia, commented on its association with sustained facial contraction, and expressed the view that, like facial palsy, it might be an early sign of multiple sclerosis. Kino (1928) reported three patients with undulating fascicular twitching of the face which he independently termed facial myokymia. All three patients were diagnosed as suffering from multiple sclerosis. Andermann et al. (1961) reviewed the literature, discussed seven previously reported cases, and added four of their own. They postulated that the myokymia resulted from a central excitatory focus of demyelination within or near the facial nucleus. However, they had no histopathological studies to substantiate their postulation.

In earlier literature there are rare references to neoplastic and vascular lesions within the pons being responsible for what was probably facial myokymia but referred to as continuous rhythmic fascicular contraction of the facial muscles, but in these cases histopathological data are meagre.

The present case is reported because of the rarity of previous reports, because the facial myokymia was associated with facial palsy, and because postmortem studies made clinicopathological correlations possible.

\footnotetext{
1 Present address: Command Hospital, Lucknow, India.
}

A 57 year old white man was admitted to hospital on 30 December 1971, suffering from productive cough, haemoptysis, and weight loss of some months' duration. He had been a heavy smoker for many years. There was no history of fever or of pains around the face.

He was oriented as to time, place, and person but confused and lethargic and unable to describe his symptoms well. There was a palsy of the left face, involving both upper and lower portions, with lagophthalmos and Bell's phenomenon. There were involuntary movements of the left facial muscles, most marked around the mouth and in the platysma, but also seen in the zygomatic region around the lower lid. The movements were continuous, wormlike, and undulating; varying in location and not affecting all areas at the same time; undisturbing to the patient; and unaffected by sleep or emotional or volitional movements of the face, jaw, or eyes, or by patting or pulling of the skin of the face. There were no percussion myotonia or myoedema, and no abnormal synkineses or associated movements. There was no tenderness around the external ear or mastoid and no hearing loss, and the external acoustic meatuses were normal.

There was also a left partial homonymous hemianopia, affecting the lower visual fields more than the upper and sparing the macular area. Otherwise cranial nerve functions were intact and the power, tone, coordination, and sensory functions in the limbs were good and equal on the two sides. The deep reflexes in the limbs were present and equal and the plantar responses normal.

The blood pressure was $120 / 80 \mathrm{mmHg}$, the pulse 100 per minute and regular. His right upper chest moved poorly on respiration and auscultation re- 
vealed rhonchi in the underlying lung and radiography showed a right upper lobe cavity.

LABORATORY DATA The sputum was repeatedly negative for acid-fast bacilli and malignant cells, and no definite pathogenic organisms were grown. Other routine laboratory tests on urine and blood, including those for syphilis, were normal.

Nerve conduction studies showed normal conduction times in the facial nerve (left orbicularis oculi$3 \mathrm{~ms}$, conduction distance being $8 \mathrm{~cm}$; left orbicularis oculi-2 ms, conduction distance being $6 \mathrm{~cm}$. Electromyography using fine monopolar needle electrodes showed a suggestion of rhythmic spontaneous discharge in the platysma, and in all other areas only spontaneous continuous low amplitude potentials, hardly distinguishable from normal motor unit action potentials except for their low amplitude.

HOSPITAL COURSE The patient became more and more lethargic and confused. Two weeks after admission he had a further bout of haemoptysis and about the same time an episode of dizziness with falling. He was disoriented as to time and place and exhibited motor impersistence in that he had to be repeatedly reminded to keep his eyes closed during sensory testing. The left facial weakness persisted but the myokymia was now limited to the platysma muscle. There was also mild weakness of the left limbs and some suggestion of his neglecting the left half of the body on bilateral simultaneous stimulation. The knee and ankle jerks were brisker on the left than on the right and there was on the left an illsustained ankle clonus and a Babinski plantar response. Pin-prick appreciation in the face and corneal sensitivity were depressed on the left. The visual field defect had not changed.

Bronchoscopy showed, coming mainly from the right bronchial trees, abundant purulent serosanguineous secretion, specimens of which failed to reveal tumour tissue.

On the electroencephalogram there was depression of background rhythm on the right hemisphere and mild to moderately severe slowing over the central and parietal areas on the right. No epileptiform discharges were seen.

Brain scan revealed independent areas of increased uptake in the right posterior frontal and right parietal region; and a right carotid arteriogram showed avascularity in the posterior frontal region and a small 'blush' in the right parietal area. There was no evidence of displacement of midline vessels or of uncal herniation.

On 16 January 1972, a large frontoparietal craniotomy revealed evidence of what to the naked eye appeared to be two tumours, one on the right inferior frontal area, and the other in the right lateral parietal area. Biopsy from these areas showed frag- $\stackrel{\mathbb{D}}{\circ}$ ments of cerebral cortex, extensive areas of necrosis, $\bar{O}$ and 'nests' of tumour cells which the pathologist thought to be of metastatic origin and probably adenocarcinomatous. Postoperatively the patient remained disoriented and lethargic and over the ensuing week deteriorated and died.

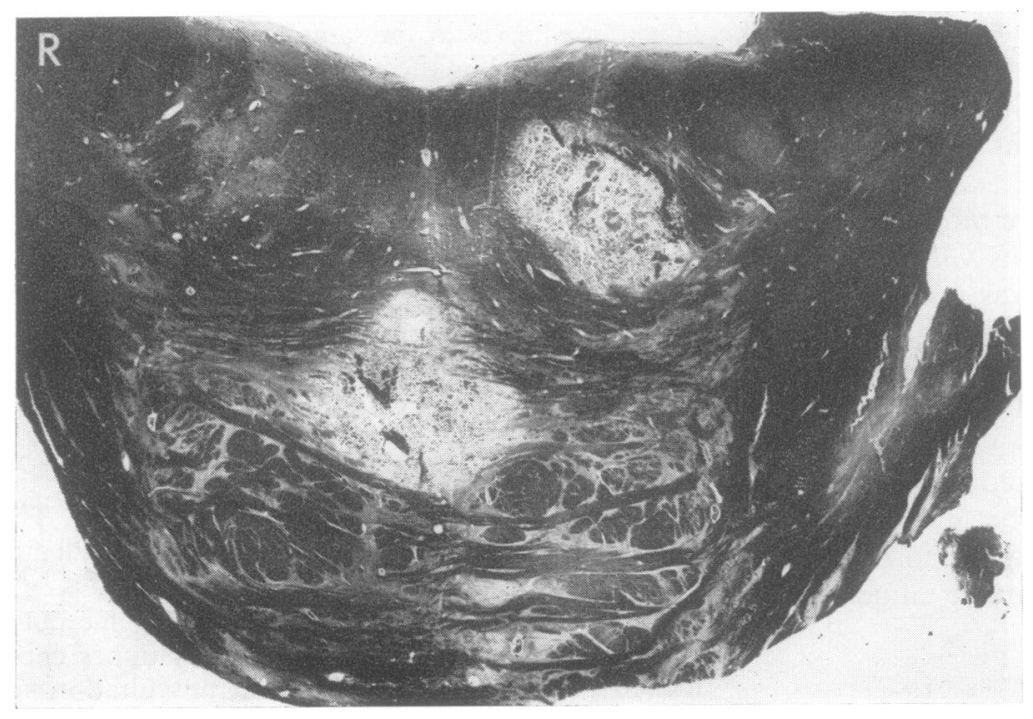

FIG. 1. Section of pons showing the two metastases, one median, the other in the left tegmentum. 
PATHOLOGICAL FINDINGS At necropsy, bronchogenic carcinoma in the upper lobe of the right lung was confirmed, with metastases to lymph nodes, liver, kidneys, and adrenal glands. The brain was somewhat swollen and its convolutions flattened and the pia-arachnoid membranes over the right lateral frontal convexity were opaque. The arteries at the base showed spotty atheromatosis without luminal occlusion.

On coronal sections, a slight general brain swelling was confirmed. In the white matter of the right posterior frontal and inferior parietal areas there were several small fine cystic areas of encephalomalacia and there was softening in the right parietal subcortical area and in the right superior temporal gyrus.

Fairly sharply delimited areas of pale grey discolouration were found in the tegmentum of the left mid pons and in the upper and mid pons on the right in the region of the medial lemniscus. No lesions were noted in the midbrain, cerebellum, or medulla, and no lesions of the facial nerve or its nucleus were detected.

Histologically no cancer cells were obvious in the necrotic tissue in the right hemisphere, but several of the pial arteries were packed with cancer cells. No gross atheromatous lesions were encountered, and the large arteries at the base were unoccluded. The cancer cell stasis in the local leptomeningeal arterioles was thought to have contributed to the underlying encephalomalacia.

The face area in the motor and sensory cortex was microscopically intact and the internal capsule showed no lesion at any level. The corticospinal and corticobulbar fibres of the midbrain were also intact.

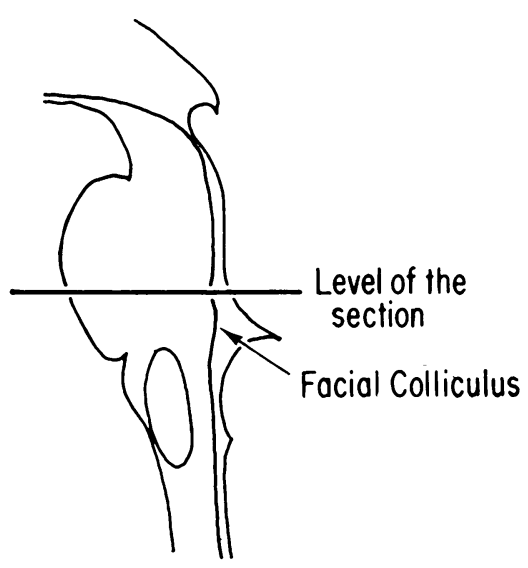

FIG. 2. Diagrammatic representation of the level of pons at which the section was taken.
In the upper and mid pons, two well-circumscribed metastases were noted, corresponding to the areas of greyish discolouration in the gross specimen (Fig. 1).

One of the metastases was in the left tegmentum, and the other in the basis pontis with greater extension to the right than the left side. These metastases appeared to be rostral to and not to involve the facial nucleus or its intramedullary fibres-the level of the lesion is indicated in Fig. 2 where the section is just above the facial colliculus.

\section{DISCUSSION}

The facial musculature may be involved in various types of involuntary movements. A careful clinical examination combined with relevant ancillary investigations can give much help in differentiating them.

Facial myokymia is one of the rare facial dyskinesias but stands out as a distinct clinical entity. It is usually easily distinguished from fasciculations, which appear as brief, irregularly occurring twitches in different parts of the facial muscles; whereas myokymia is a slower contraction involving small bands or strips of muscles and occurs more continuously, transmitting a ripple (like a 'bag of worms') to the overlying skin.

Benign myokymia of normal people is limited to the eyelids and tends to occur during states of fatigue.

The facial myokymia under discussion has been reported so far in only 45 patients. Noteworthy among previous reports is that of Andermann and his colleagues (1961) who described four cases of their own and culled seven from the literature. Matthews (1966) described a further six personal cases, and Gutmann et al. (1969) described two more and gathered 40 from the literature. Most of these cases had been found to be due to multiple sclerosis; but Espinosa et al. (1967) found evidence to incriminate tumours in and around the pons. Of their patients with facial myokymia, 10 had clinical evidence of intramedullary tumour of the brain-stem, and two had extramedullary tumours of the posterior fossa. Of the 10 patients considered to have had intramedullary tumours, the clinical diagnosis was strengthened in four cases by the findings on pneumoencephalography; of the other six patients, one had glioma of the brain-stem and cerebellum, and the others had biopsy evidence 
of astrocytomas of the pons. Detailed histopathological studies with a view to localization are not available.

Two facts emerge from the reports of facial myokymia in multiple sclerosis: the myokymia, although it may recur in the same or the opposite side of the face, is almost invariably transient (persisting from two days to three months, with the exception of one case in which it lasted six months); and appreciable facial paresis has not been a feature. In contrast, in the cases associated with tumours (Espinosa et al., 1967) significant and progressive facial paralysis was often present as well as progressive involvement of other cranial nerves and of the pyramidal and cerebellar systems.

There have been no previous reports incriminating metastatic lesions. In our case the pontine metastases were thought to be the cause of the

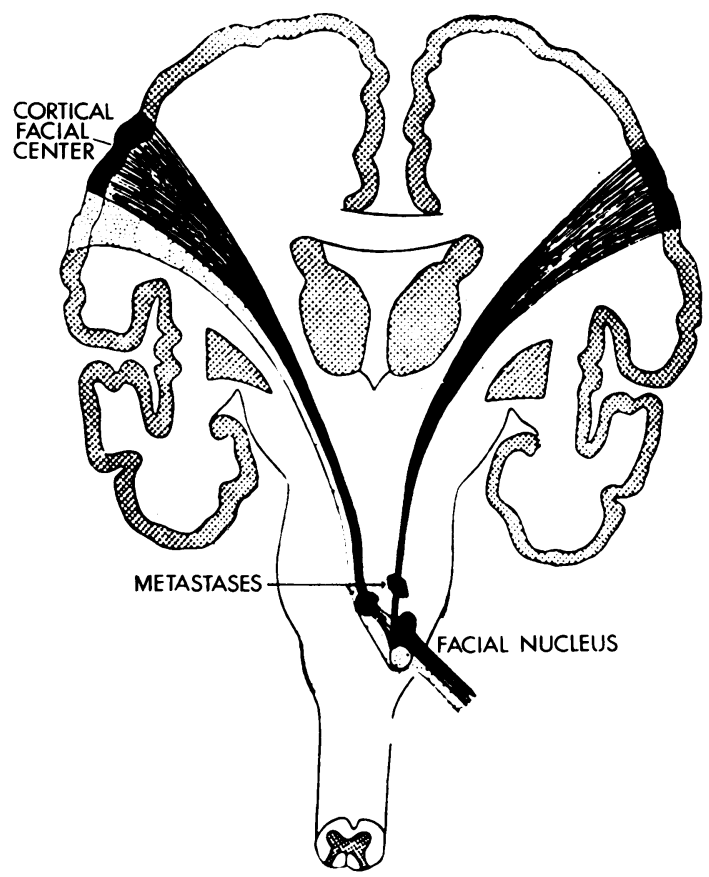

FIG. 3. Diagrammatic representation of crossed and uncrossed corticobulbar fibres as they approach and enter facial nucleus. (Modified from Bing's Local Diagnosis in Neurological Diseases.) Approximate location of metastases is also diagrammatically shown. $\square=$ To upper part of face. . of face. myokymia and the dense paralysis affecting the upper and lower left face; the lesions in the right posterior frontal, temporal, and parietal regions were thought to explain the progressive left hemiparesis, homonymous hemianopia, and left-sided sensory inattention.

No lesion was found that might involve the peripheral part of the facial nerve; and the continuing normal conduction velocities in that nerve also helped to exclude such a lesion. Nor was there macroscopic or microscopic evidence that the facial nucleus was involved, so a nuclear lesion can be excluded as the cause of the myokymia. Likewise, the lesions were too rostral to involve the intramedullary portion of the seventh nerve; nor was there clinical evidence of sixth nerve palsy or of a defect of conjugate lateral gaze such as often accompanies such an intramedullary facial nerve paralysis. In brief, the clinical and pathological findings favoured a supranuclear lesion of the left facial neurones.

There appear to be no universally agreed data as to the course of the corticobulbar fibres below the level of the internal capsule. Dejerine's view (1906), based on human material, was that the corticobulbar fibres were subdivided into groups, some running directly to their termination in close association with the corticospinal (pyramidal) fibres; other (so-called aberrant) fibres leaving the corticospinal tract at various levels in the midbrain and pons to pass to their respective cranial motor nuclei. Decussation of the corticobulbar fibres is partial and occurs near the level of the cranial motor nuclei for which they are destined.

Kuypers (1958) has described direct and indirect corticobulbar fibres in man. The facial (and hypoglossal) nuclei receive fibres from both corticobulbar systems, direct and aberrant. The corticobulbar fibres arise in the precentral gyrus and in the adjoining middle and inferior prefrontal gyri, traverse the internal capsule near its genu, and pass through the basis pedunculi in its middle three-fifths in association with the corticospinal fibres to enter the pons. In the pons, near the level of the seventh motor nucleus, a group of fibres breaks away and passes dorsally into the tegmentum where some decussate to terminate in the contralateral facial motor nucleus, especially in the part of the nucleus which innervates the lower facial muscles. 
Some decussating fibres proceed to cells that innervate the upper facial muscles (frontalis, orbicularis oculi and corrugator supercilii): these muscles are also supplied by non-decussating fibres from the ipsilateral hemisphere. In brief, the upper face has bilateral, and the lower face mainly unilateral representation. This view is also supported by electrical stimulation studies on the human cortex (Foerster, 1936; Penfield and Boldrey, 1937) and, of course, by clinical data.

Figure 3 is a diagrammatic representation of the corticobulbar fibres as they approach and enter the facial nucleus (directly or through intercalated cells). The two pontine metastases are in a position to involve these fibres, the more central one interrupting the crossing fibres for the left lower and upper face, the other interrupting ipsilateral fibres for the left upper face. In other words, the two lesions in combination are interrupting all the main supranuclear pathways for the control of the left upper and lower face.

Although the metastases do not involve the facial nucleus directly, they are perhaps sufficiently near it (1) to set up the steady depolarizing state postulated by Andermann and his colleagues as affecting the soma-dendritic membranes of the facial motor neurones and so causing the facial myokymia; or, alternatively (2) to 'release' the facial nucleus from higher control, resulting in increased excitability of the facial motor neurones.

In further support of the causative lesion of myokymia being rostral to the seventh nerve and its nucleus was the observation by Andermann and his co-workers (1961) that two of their patients had trigeminal nerve involvement, and the similar finding in two of Matthews's (1966) patients.

Because in our patient there was paralysis of both upper and lower facial muscles, we naturally considered the likelihood of a lower motor neurone lesion. We finally concluded that the left facial palsy was of central type and that the explanation for the upper and lower facial palsy lay in the fact that all the supranuclear connections to the left facial muscles, both contralateral and ipsilateral, had been interrupted.

We are most grateful to Dr. Joseph C. Lee for his valuable suggestions and criticism. We also appreciate the courtesy of Dr. Webb Haymaker and the publishers, The C.U. Mosby Co, St. Louis, for permission to modify Fig. 14-5 in the 15th edition of Bing's Local Diagnosis in Neurological Diseases, ed. 15 (1969).

\section{REFERENCES}

Andermann, F., Cosgrove, J. B. R., Lloyd-Smith, D. L,. Gloor, P., and McNaughton, F. L. (1961). Facial myokymia in multiple sclerosis. Brain, 84, 31-44.

Dejerine, J. (1906). L'aphasie sensorielle: sa localisation et sa physiologie pathologique. Presse Médicale, 14, 437-439; 453-457.

Espinosa, R. E., Lambert, E. H., and Klass, D. W. (1967). Facial myokymia affecting the electroencephalogram. Mayo Clinic Proceedings, 42, 258-270.

Foerster, O. (1936). Motorische Felder und Bahnen, in Handbuch der Neurologie, Vol. 6, pp. 1-357. Edited by 0. Bumke and O. Foerster.

Gutmann, L., Thompson, H. G., Jr., and Martin, J. D. (1969). Transient facial myokymia. Journal of the American Medical Association, 209, 389-391.

Kino, F. (1928). Muskelwogen (Myokymie) als Frühsymptom der multiplen sklerose. Deutsche Zeitschrift für Nervenheilkunde, 104, 31-41.

Kuypers, H. G. J. M. (1958). Corticobulbar connexions to the pons and lower brain-stem in man. Brain, 81, 364-388.

Matthews, W. B. (1966). Facial myokymia. Journal of Neurology, Neurosurgery, and Psychiatry, 29, 35-39.

Oppenheim, H. (1916). Leistungen und Fortschritte aus dem Gebiete der Neurologie und Psychiatrie, 20, xiv (cited by Andermann et al., 1961).

Penfield, W., and Boldrey, E. (1937). Somatic motor and sensory representation in the cerebral cortex of man as studied by electrical stimulation. Brain, 60, 389-443. 